\title{
REFLECTIVITY STUDIES OF LATTICE VIBRATIONS AND FREE ELECTRONS IN MBE GROWN GaN EPITAXIAL LAYERS
}

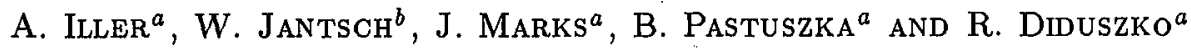 \\ ${ }^{a}$ Institute of Vacuum Technology, Długa 44/50, 00-241 Warszawa, Poland \\ ${ }^{b}$ Institut für Experimentalphysik, Johannes Kepler Universität \\ Altenbergerstr. 69, $4040 \mathrm{Linz}$, Austria
}

\begin{abstract}
We have observed a sharp structure with a peak at the frequency of the $E_{1}$-TO phonon in the reflectivity of GaN epitaxial layers grown by molecular beam epitaxy on Si substrates. The simulations of the reflection performed show that the observed shape can be explained by assuming both collective lattice vibrations and free carriers contributions to the dielectric function. We assumed the Lorentz oscillator to describe the contribution of the collective lattice vibrations and the Drude-Lorentz model for that of free carriers. Fitting the calculated reflectivity to the spectrum obtained experimentally allowed us to evaluate lattice and free carrier parameters.
\end{abstract}

PACS numbers: 63.20.-e, 78.66.Fd

\section{Introduction}

Several authors have investigated infrared reflectivity in GaN. Only a vibrational band in the spectral range from $500 \mathrm{~cm}^{-1}$ to $800 \mathrm{~cm}^{-1}$ was observed for epitaxial layers with carrier concentration below $10^{18} \mathrm{~cm}^{-3}[1,2]$. An analysis based on the dielectric function of the form of the dielectric oscillator allowed us to determine the transverse and longitudinal optical mode frequencies, the high-frequency dielectric constant, the oscillator strength and damping constant of the transverse optical mode. The plasma edge was found in the reflectivity spectrum of bulk crystals with an electron concentration exceeding $6 \times 10^{19} \mathrm{~cm}^{-3}$ in the 1000-3000 $\mathrm{cm}^{-1}$ region [3]. The determination of the plasma frequency enabled the authors to evaluate the ratio of the free carrier concentration and the effective mass. Reflectivity studies were reported for samples with free carrier concentration in the $2 \times 10^{17}-1 \times 10^{20} \mathrm{~cm}^{-3}$ range by Barker et al. [2] and in the $10^{19} \mathrm{~cm}^{-3}$ range by Manchon et al. [4]. The correlation of the reflectivity spectrum with the results of Hall measurements allowed Barker et al. to determine the value of the optical effective mass. The analysis performed by Manchon et al. resulted in determination of infrared phonon and electron parameters. 
We report here measurements of the infrared reflectivity of molecular beam epitaxy (MBE) grown epitaxial layers in $10 \mathrm{~cm}^{-1}$ to $5000 \mathrm{~cm}^{-1}$ region. The aim of the present paper is to explain the spectra obtained and to deduce the parameters describing phonons and free carriers separately.

\section{Growth procedure and experiment}

GaN epilayers were grown by MBE in an MBE-01 machine designed and manufactured in the Institute of Vacuum Technology. The technological chamber of the machine is equipped with gallium and aluminium effusion cells, a compact ECR source (from ASTEX) fed with ultrahigh purity $\mathrm{N}_{2}$ for atomic nitrogen generation and growth control system containing the reflection high-energy electron diffraction (RHEED) and a quadrupole mass spectrometer. During growth, the system is pumped using cryo- and turbomolecular pumps.

The nitride layers have been grown on two kinds of substrates: $\mathrm{Si}(111): \mathrm{P}$ $(\rho=200 \Omega \mathrm{cm})$ or $\mathrm{Si}(001)$ :As $(\rho=0.005 \Omega \mathrm{cm})$. All the substrates were cleaned in a normal Shiraki process [5] with HF passivation and mounted with indium on Mo sample holder. In order to desorb native oxides the substrates were annealed in the deposition chamber at $900^{\circ} \mathrm{C}$ for $30 \mathrm{~min}$ immediately before the growth process. In some cases, a thin AlN film was deposited as a buffer layer before growing the GaN layer.

The AlN buffer layers have been grown at the substrate temperature of $700^{\circ} \mathrm{C}$ with a growth rate of about $60 \mathrm{~nm} / \mathrm{h}$ to a thickness of about $20 \mathrm{~nm}$. The GaN layers were deposited with a growth rate of about $0.1 \mu \mathrm{m} / \mathrm{h}$ at $650^{\circ} \mathrm{C}$ substrate temperature.

The layer thicknesses were in the range of 75 to $285 \mathrm{~nm}$. The crystallographic quality of the layers and their orientation were investigated in situ by RHEED and post growth by X-ray diffraction. X-ray diffraction measurements in the $\omega / 2 \theta$ as well as the $\omega$ mode were made using a Siemens D500 diffractometer with Cu $K_{\beta}$ radiation. The layers have the hexagonal, wurtzite-type structure with their $c$-axis oriented perpendicularly to the layer surface, independent of the substrate orientation. The structural quality is improving with increasing thickness. The structural parameters determined for the thickest layer $(285 \mathrm{~nm})$ are $c=0.5182 \mathrm{~nm}$, the FWHM for an $\omega$ scan is $143^{\prime}$, and FWHM for an $\omega / 2 \theta$ scan is $5.7^{\prime}$.

Infrared measurements in reflection geometry at nearly normal incidence were taken using a Fourier transform infrared spectrometer, Beckman IR-720 from $80 \mathrm{~cm}^{-1}$ to $680 \mathrm{~cm}^{-1}$, and Nicolet IMX-10 from $500 \mathrm{~cm}^{-1}$ to $4000 \mathrm{~cm}^{-1}$. The spectra measured in both spectral ranges were combined in one spectrum by normalization in the overlap common region. All the spectra were normalised to the reflection of an Al mirror. The measurements have been performed at room temperature without intentional light polarization.

\section{Results and discussion}

The reflectivity was measured for three samples, two of them (GN44 and GN45) being grown on Si (111) with AlN buffer layer and one (GN46) - directly on $\mathrm{Si}(100)$. The spectra for the samples GN45 and GN46 are presented in Fig. 1 together with the results of Barker et al. [2] and Manchon et al. [4]. 
The spectrum for the sample GN44 is similar to that for the sample GN45 and is not shown for clarity in the figure. The measurements of Barker et al. have been made on single-crystal epitaxial layers on (0001) $\alpha-\mathrm{Al}_{2} \mathrm{O}_{3}$ crystals. Stars represent the reflectivity of a sample for which the carrier concentration was reduced below $10^{18} \mathrm{~cm}^{-3}$ by bombardment by $1 \mathrm{MeV}$ electrons. The sample represented by diamonds was not bombarded. The sample of Manchon et al. was composed from six hexagonal needles and then mechanically polished. The concentration obtained from the Hall measurement made on a crystal from a similar growth batch was $3 \times 10^{19} \mathrm{~cm}^{-3}$. It is seen that our result differs from all other results reported in the literature.

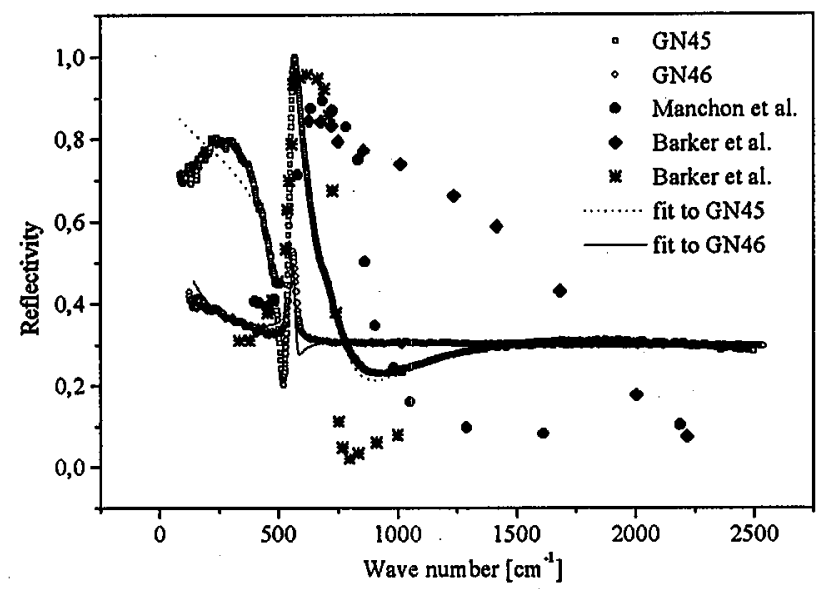

Fig. 1. Infrared reflectivity spectrum of GaN. The open circles and squares give our experimental data for the samples GN45 and GN46, respectively. The stars and solid diamonds are Barker's data after electron bombardment to remove free carriers and those of conducting samples, respectively. The curves are fits using one lattice oscillator plus a plasma term.

In order to understand the obtained experimental results the calculations of the reflectivity have been performed. The complex dielectric function in the form

$$
\varepsilon(\omega)=\varepsilon_{\infty}+\chi_{\mathrm{SN}}(\omega)+\chi_{\mathrm{S}}(\omega)
$$

has been assumed, where $\chi_{\mathrm{SN}}(\omega)$ and $\chi_{\mathrm{S}}(\omega)$ are susceptibility contributions due to free carriers and lattice vibrations, respectively and $\varepsilon_{\infty}$ is a constant which accounts for the contribution of valence electrons. The lattice vibration contribution term has been assumed in the form of one Lorentzian oscillator [6]

$$
\chi_{\mathrm{S}}(\omega)=\frac{S \omega^{2}}{\omega_{\text {TO }}^{2}-\omega^{2}-\mathrm{i} \Gamma \omega} .
$$

The resonance frequency $\omega_{\text {TO }}$ of the oscillator is equal to the frequency of the $E_{1}$ transverse optical phonon, $S$ is the oscillator strength, and $\Gamma$ is a phenomenological parameter describing the system damping. In the geometry $\boldsymbol{E} \perp \boldsymbol{c}$ of our 
experiment the second infrared active mode, $A_{1}$, could not be excited. For the free carrier contribution term in formula (1) the Drude-Lorentz model has been assumed

$$
\chi_{\mathrm{SN}}(\omega)=\frac{\omega_{\mathrm{p}}^{2}}{-\omega^{2}-\mathrm{i} \omega / \tau},
$$

where the plasma frequency $\omega_{\mathrm{p}}$ is determined by the free carrier concentration $N$ and their effective mass $m^{*}: \omega_{\mathrm{p}}=\sqrt{N e^{2} / \varepsilon_{0} m^{*}}$ and the relaxation time $\tau$ defines free carrier damping. In order to calculate the reflectivity, the formula

$$
R=\frac{(n-1)^{2}+k^{2}}{(n+1)^{2}+k^{2}}
$$

for an infinite medium has been applied, which is justified by the flat reflectivity spectrum of the Si substrate. Interference effects also do not affect the reflectivity in the spectral region and layer thickness under consideration. In the relation (4) the refraction coefficient $n$ and the extinction coefficient $k$ are the real and imaginary part of the complex refractive index, respectively.

TABLE

Lattice and free carrier parameters of $\mathrm{GaN}$ obtained from the fitting of classical theory of dielectric function to infrared reflectivity.

\begin{tabular}{c|c|c|c|c}
\hline \hline Parameter & GN44 & GN45 & GN46 & Other papers \\
\hline Substrate & AlN/Si(111) & AlN/Si(111) & Si(100) & \\
\hline $\begin{array}{c}\omega_{\text {To }}\left[\mathrm{cm}^{-1}\right] \\
E_{1} \text { mode }\end{array}$ & $561 \pm 3$ & $557 \pm 0.3$ & $559 \pm 77$ & $\begin{array}{c}565 \pm 2[1], \\
560 \pm 1 \%[2], \\
\end{array}$ \\
& & & $\begin{array}{c}559[4],[7]^{*},[8]^{*}, \\
561[9]^{*},[10]^{*}\end{array}$ \\
\hline$\omega_{\mathrm{p}}\left[\mathrm{cm}^{-1}\right]$ & $714 \pm 23$ & $749 \pm 3$ & $883 \pm 42$ & $4800[2]$ \\
\hline $1 / \tau\left[\mathrm{cm}^{-1}\right]$ & $482 \pm 47$ & $388 \pm 5$ & $4193 \pm 440$ & $700[2]$ \\
\hline$N\left[\mathrm{~cm}^{-3}\right]$ & $7.5 \pm 0.5$ & $7.9 \pm 0.5$ & $10 \pm 1$ & $10^{12}-10^{13}[1]^{\dagger}$, \\
& $\times 10^{18}$ & $\times 10^{18}$ & $\times 10^{18}$ & $\approx 10^{19}[2]^{\dagger}$, \\
& & & & $3 \times 10^{19}[4]^{\dagger}$ \\
\hline$\mu\left[\mathrm{cm}^{2} /(\mathrm{V} \mathrm{s})\right]$ & $90 \pm 8$ & $110 \pm 1.5$ & $10 \pm 1$ & $80[2]^{\dagger}, 85[4]^{\dagger}$ \\
\hline Thickness [nm] & 285 & 190 & 75 & \\
\hline
\end{tabular}

*from Raman scattering, ${ }^{\dagger}$ from Hall measurements.

A fit of the reflectivity calculated according to the relations (1)-(4) to the experimental spectra is shown in Fig. 1. The fitting parameters are summarised in Table together with the concentration and mobility calculated from the plasma frequency and relaxation time, respectively. The value $0.22 m_{0}$ determined by Perlin et al. [3] for the effective mass has been used in the calculations. The parameters reported by other authors are also given. The values of carrier parameters for two layers grown on $\mathrm{Si}(111)$ with $\mathrm{AlN}$ buffer layer are similar. The layer grown directly on $\mathrm{Si}(100)$ has a higher carrier concentration and by one order of magnitude lower carrier mobility. This result may be explained by a large lattice mismatch between 
GaN and $\mathrm{Si}(17 \%)$, which causes a poor crystalline quality, a source of carriers and scattering centres. In the case of GaN layers grown on AlN buffer the crystalline quality is much improved. The frequency of the transverse $E_{1}$-mode optical phonon obtained by the fitting agrees very well with the values reported in the literature. Also the higher value for carrier mobility determined is in good agreement with the values obtained by other authors from Hall measurements for thick layers or bulk-like GaN samples with carrier concentration in the same range. It was not possible to measure the Hall voltage for our samples.

A much higher carrier concentration and a lower mobility can probably explain the different shape of Barker et al. spectrum for the sample that was not bombarded (diamonds in Fig. 1). Assuming $m^{*}=0.22 m_{0}$, the concentration $3.4 \times 10^{20} \mathrm{~cm}^{-3}$ and the mobility $60 \mathrm{~cm}^{2} /(\mathrm{V} \mathrm{s})$ can be obtained from Barker's data. From the data of Manchon et al. the concentration $1.4 \times 10^{19}$ can be deduced, assuming $m^{*}=0.22 m_{0}$. This result and the shape of the corresponding spectrum are not consistent with our results.

In summary, we have explained the reflectivity spectrum obtained for ECR MBE grown GaN layers in the Restrahlen region by the presence of free carriers. The carrier concentration and mobility have been evaluated from the fitting based on the classical theory of the complex dielectric function to the reflectivity spectra. The layers grown on AlN buffer layer have a lower carrier concentration and by one order of magnitude higher carrier mobility than the layer grown without AlN buffer layer.

\section{Acknowledgment}

We would like to express our gratitude to Prof. T. Dietl and Dr. A. Witowski for helpful discussions. This work was partly supported by the Committee for Scientific Research (Poland) grant No. 8T11B01909.

\section{References}

[1] H. Sobotta, H. Neumann, R. Franzheld, W. Seifert, Phys. Status Solidi B 174, K57 (1992).

[2] A.S. Barker, Jr., M. Ilegems, Phys. Rev. B 7, 743 (1973).

[3] P. Perlin, E. Litwin-Staszewska, B. Suchanek, W. Knap, J. Camassel, T. Suski, R. Piotrzkowski, I. Grzegory, S. Porowski, E. Kamińska, J.C. Chervin, Appl. Phys. Lett. 68, 1114 (1996).

[4] D.D. Manchon, Jr., A.S. Barker, Jr., P.J. Dean, R.B. Zetterstrom, Solid State Commun. 8, 1227 (1970).

[5] A. Ishizaka, Y. Shiraki, J. Electrochem. Soc. 133, 666 (1986).

[6] D. Palik, Handbook of Optical Constants of Solids II, Academic Press, New York 1991.

[7] V. Lemos, C.A. Argüello, R.C. Leite, Solid State Commun. 11, 1351 (1972).

[8] A. Cingolani, M. Ferrara, M. Lugara, G. Scamarcio, Solid State Commun. 58, 823 (1986).

[9] K. Hayashi, K. Itoh, N. Sawasaki, I. Akasaki, Solid State Commun. 77, 115 (1991).

[10] T. Azuhata, T. Sota, K. Suzuki, S. Nakamura, J. Phys., Condens. Matter 7, L129 (1995). 